\title{
A mysterious case of an elevated dome of the right diaphragm
}

A 63-year-old, nonsmoking man presented with dry cough and fever of 2 days' duration. On enquiry, he also complained of dyspnoea on exertion of modified Medical Research Council grade 1 for 2 months. There were no other respiratory complaints. He was diagnosed with a progressive supranuclear palsy-type parkinsonian disorder 2 years ago. He was taking levodopa (100 mg) plus carbidopa (25 mg) thrice daily for that condition. There was no significant occupational history, family history or similar illness in the past. He was conscious and oriented with a pulse of 84 per min, blood pressure of $124 / 76 \mathrm{mmHg}$, respiratory rate of 16 per min and oxygen saturation of $96 \%$. A respiratory system examination revealed dull notes and reduced breath sounds in the right basal region. Upon neurological examination, he had a mask-like face and speech abnormality. Cranial nerve examination was normal. A motor function examination revealed resting tremor, axial rigidity and asymmetric bradykinesia (greater on the right than on the left). His gait was normal and his sensory functions were intact. The routine biochemical and haematological investigations were normal. The chest radiograph of the patient is shown in figure 1. It shows an elevated right dome of the diaphragm, a patchy opacity near the right costophrenic angle and a doubtful opacity near the right cardiophrenic angle. The right hilum was not visualised. There was no history of chest radiography performed in the past. Ultrasound

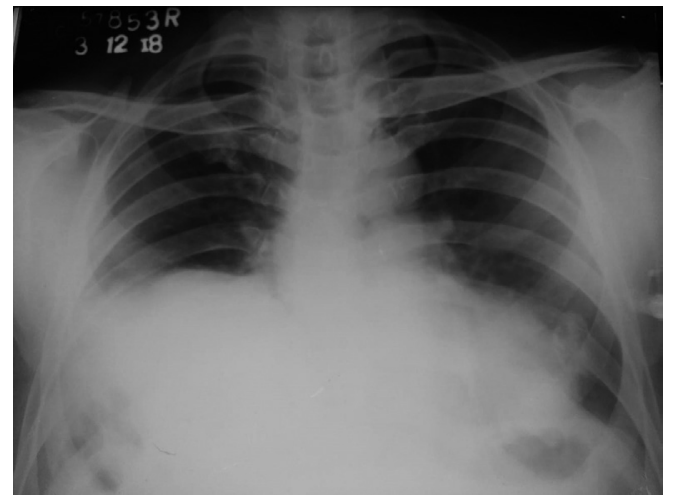

Figure 1 Chest radiograph of the patient in posteroanterior projection.

revealed an elevated position of liver but there was no evidence of pleural effusion or hepatic abnormality. The diaphragmatic movement and thickness were assessed using ultrasound $M$ and $B$ modes, respectively. The excursion of the right diaphragm with respiration was similar to that of the left diaphragm during quiet breathing. A sniff test was also normal. The muscle thickness of the right and left diaphragm was $2.4 \mathrm{~mm}$ and $2.5 \mathrm{~mm}$, respectively, at functional residual capacity in the zone of apposition. The thickness increased by $>20 \%$ with inspiration. A computed tomography (CT) scan of the thorax with contrast study images are shown in figures 2 and 3.
Cite as: Gothi D, Patro M Agarwal $M$, et al. A mysterious case of an elevated dome of the right diaphragm. Breathe 2020; 16: 190334. 

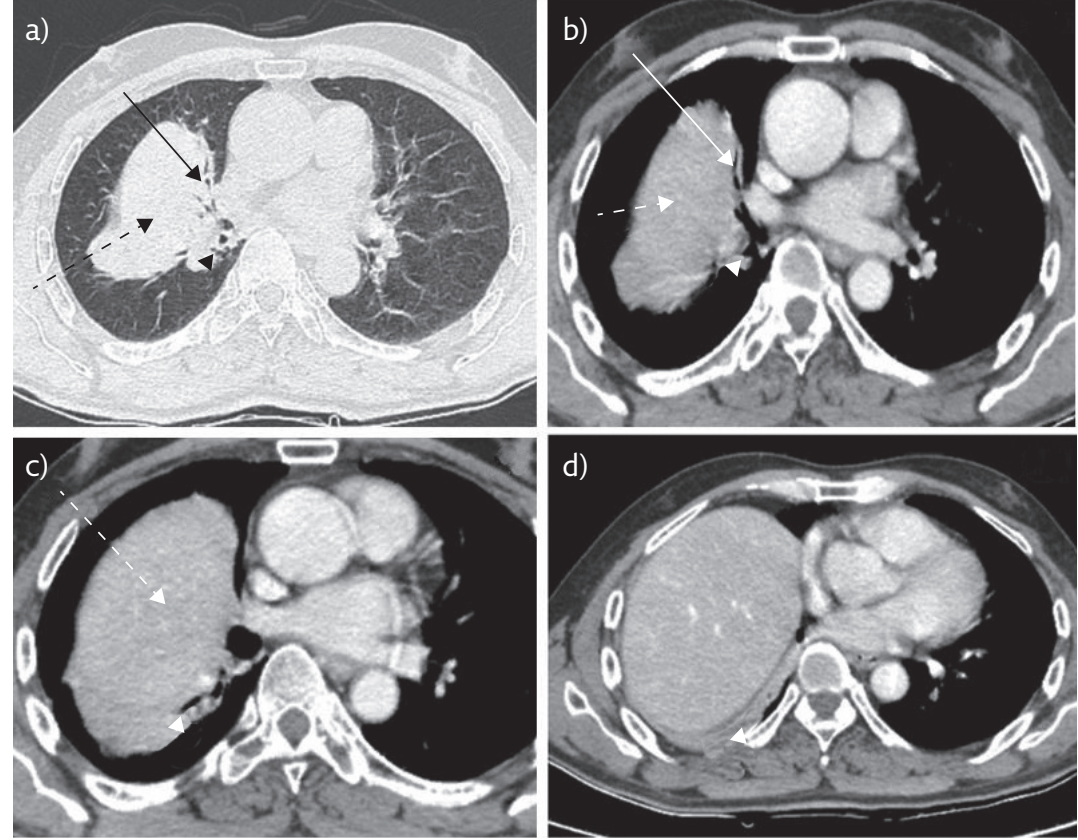

Figure 2 CT of the chest: a) axial section of the lung window; $b$-d) axial sections of the mediastinal window at different levels of cross-section. The abnormalities are marked with arrows, arrowheads and dashed arrows; the key to which is provided answer 1.

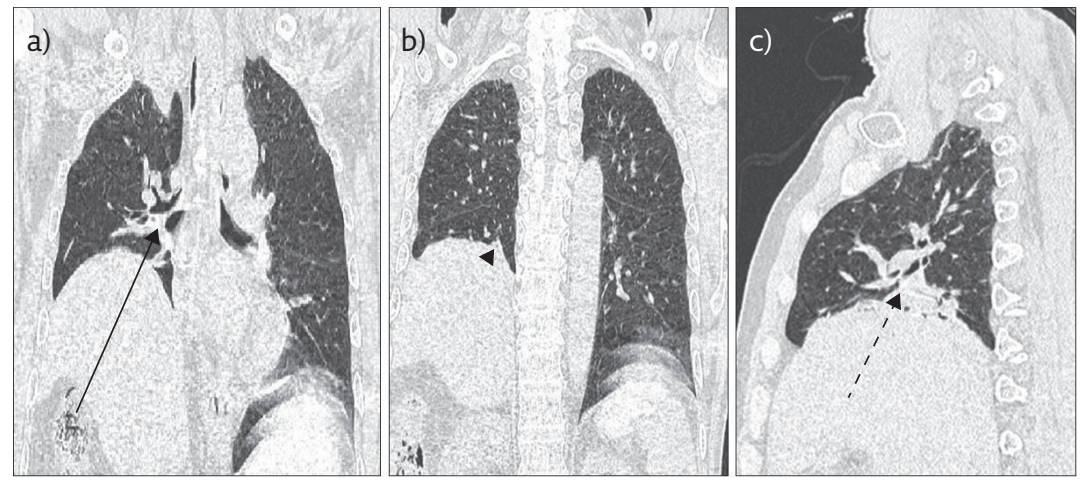

Figure 3 CT of the chest: $a$ and $b$ ) coronal sections of the lung window; c) sagittal section of the lung window. The abnormalities are marked with arrows, arrowheads and dashed arrow, the key to which is provided in answer 1 .

\section{Answer 1}

$\mathrm{b}$ and $\mathrm{c}$. Right middle and right lower lobe collapse

The CT scan is suggestive of combined collapse of the right middle lobe and right lower lobe. The abnormalities are marked with arrows. Figure 2 represents the axial sections of the $C T$ scan in the lung and mediastinal windows showing right middle lobe collapse (arrow) and lower lobe collapse (arrowhead) with an elevated dome of the diaphragm (dashed arrow). Figure 3, i.e. the sagittal and coronal sections in the lung window, also shows right middle lobe collapse (arrow), lower lobe collapse (arrowhead), and middle and lower lobe combined collapse (dashed arrow).

The combined middle and lower lobe collapse or atelectasis can be difficult to detect on a chest radiograph. The diagnosis should be suspected in patients with a small right hilum or an oligaemic hyperinflated right upper lobe and elevated diaphragm. Sometimes, a combined collapse mimics a lower lobe collapse. Due to these unusual features, the combined collapse requires $\mathrm{CT}$ scan for confirmation [1].

\section{Task 2}

What could be the cause of collapse in this case?

a. Foreign body obstruction

b. Malignant or benign tumour

c. Inflammatory stricture

d. Mucous plug occluding bronchus intermedius

e. Cannot be ascertained

\section{Task 1}

Which of the follow are the diagnosis based on the radiological findings?
a. Right-sided subpulmonic effusion
b. Right middle lobe collapse
c. Right lower lobe collapse
d. Right diaphragmatic eventration
e. Paralysis of right diaphragm 


\section{Answer 2}

e. Cannot be ascertained

The bronchus intermedius is the common pathway to the right middle and lower lobe. A single localised lesion involving the bronchus intermedius may give rise to combined atelectasis of these lobes. The common causes of combined right middle and lower lobe collapse are tumour, foreign body, mucous plug and inflammatory stricture [1]. On the CT scan, there was no visible mass lesion, so a bronchogenic carcinoma or benign tumour was unlikely but an endobronchial lesion still can be a possibility. The patient did not have any history of foreign body aspiration. The presence of an inflammatory stricture or mucous plug cannot be confidently ruled out based on CT scan alone.

The next investigation for identification of the cause is a direct bronchoscopic visualisation of the tracheobronchial tree. Our patient was subjected to fibreoptic bronchoscopy. The bronchoscopic images are shown in figure 4.

\section{Task 3}

What is the diagnosis based on bronchoscopic images?

a. Crescent-type tracheobronchomalacia (TBM)

b. Sabre sheath-type TBM

c. Circumferential-type TBM

d. Excessive dynamic airway collapse (EDAC)

\section{Answer 3}

d. Excessive dynamic airway collapse

The bronchoscopic images in figure 4 show that there is collapsibility of the posterior wall of the trachea (figure $4 a$ and $b$ ) and bronchus intermedius (figure $4 c$ and $d$ ) on expiration. The collapse of trachea is $>70 \%$ and that of bronchus intermedius is almost complete on exhalation.

The collapse of the intrathoracic trachea and main stem bronchi is called expiratory central airway collapse (ECAC). ECAC has been further classified according to the morphology on inspiration and expiration into four different subtypes (figure 5) [2-5]:

- crescent-type TBM

- sabre sheath-type TBM

- circumferential-type TBM

- EDAC

The crescent-type TBM is characterised by weakness and collapse of the anterior tracheobronchial cartilage wall with or without invagination of the posterior membranous wall; it is usually seen in a tracheobronchomegaly [3]. The sabre sheath-type TBM seen in COPD is characterised by lateral wall collapse. The circumferential-type TBM, typically seen in relapsing polychondritis, shows the collapse of both the anterior and lateral cartilaginous wall on bronchoscopy [2]. EDAC is a condition characterised by inward bulging of atrophic muscle fibres in the posterior airway membrane during exhalation [2]. Our patient had EDAC, suggesting atrophic muscle of the membranous part of the posterior tracheobronchial wall. EDAC has been rarely reported as a cause of lobar collapse [6, 7].

Thus, the final diagnosis in our case was a combined right middle and lower lobe collapse due to EDAC in a case of a progressive supranuclear palsy-type parkinsonian disorder.

\section{Task 4}

What additional investigations will be helpful in the evaluation of ECAC?
a. Dynamic inspiratory and expiratory CT scan
b. Virtual bronchoscopy
c. Spirometry with flow-volume loop
d. All the above 


\section{Answer 4}

d. All the above

All the investigations in task 4 serve as adjuncts in the diagnostic evaluation of ECAC. The dynamic CT scan during inspiration and expiration can identify the changes in airway calibre with the phase of respiration. It is a highly sensitive method for detecting airway malacia and has been shown to be concordant with dynamic flexible bronchoscopy [8].
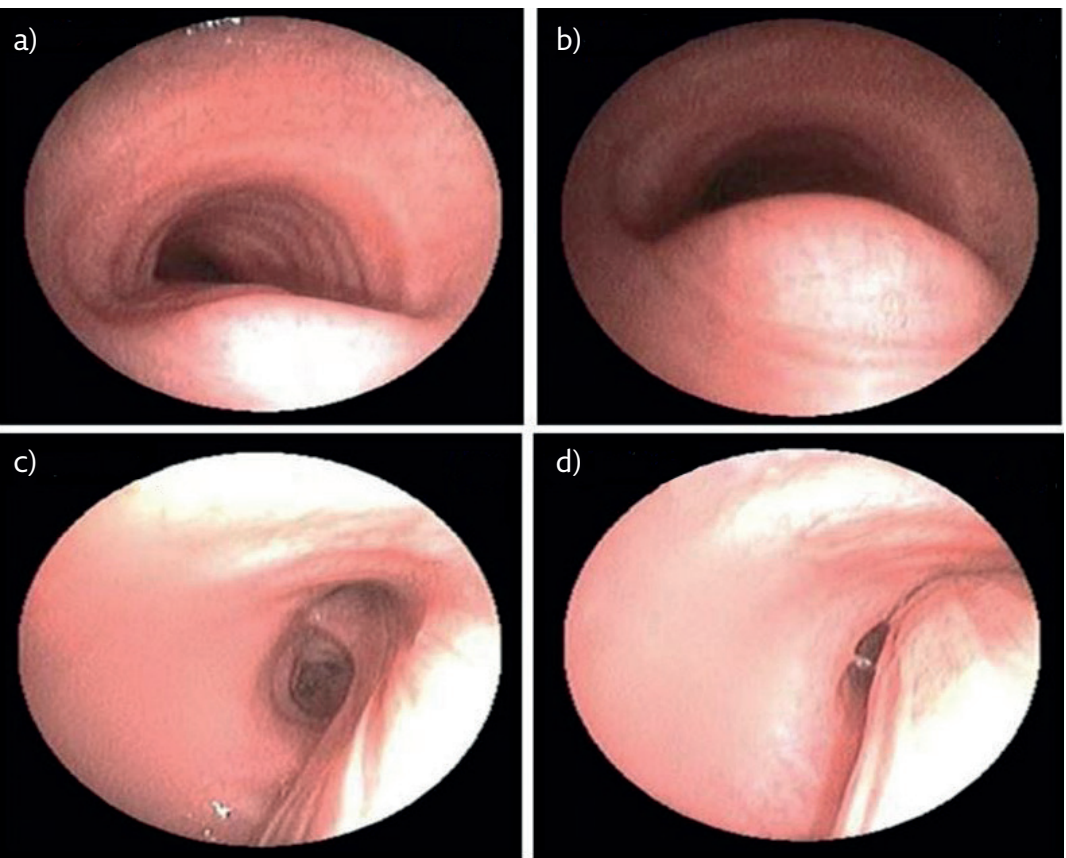

Figure 4 Bronchoscopic images: trachea during a) inspiration and b) expiration; bronchus intermedius during c) inspiration and d) expiration. a)

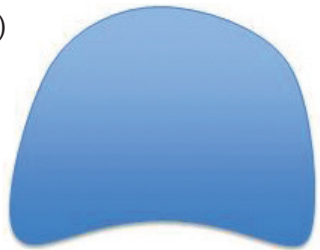

c)

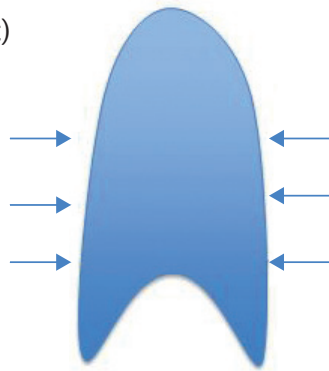

b)

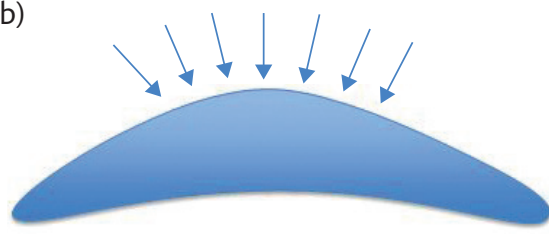

d)

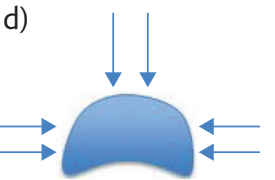

e)

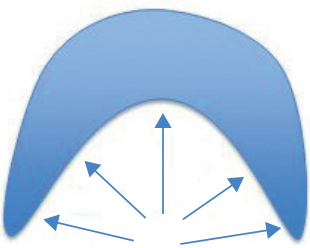

Figure 5 Various subtypes of excessive central airway obstruction: a) normal shape of trachea; b) crescent-type tracheobronchomalacia (TBM); c) sabre sheath-type TBM; d) circumferential TBM; e) excessive dynamic airway collapse.
Virtual bronchoscopy refers to the representative images of the tracheobronchial tree created by information derived from imaging sources other than bronchoscopy. Recent developments in CT with multiplanar image reconstruction and virtual bronchoscopy can depict the three-dimensional extent of involvement of the airways, which can detect ECAC [8]. Though it cannot replace fibreoptic bronchoscopy as the gold standard for detecting tracheobronchomalacia, it can be considered as an alternative diagnostic modality in patients with symptoms suggestive of tracheobronchomalacia where bronchoscopy is unavailable.

Spirometry may show an obstructive pattern with evidence of upper airway obstruction on the flow-volume loop. These findings are neither sensitive nor specific for the diagnosis of ECAC, but support the diagnosis among symptomatic patients. A substantial number of patients (21\%) with moderate to severe ECAC can demonstrate normal flow-volume loops [9]. Our patient too had normal flow-volume loop (figure 6). Table 1 shows the patient's spirometric findings. The indices of upper airway obstruction, i.e. Empey's index and the Miller and Hyatt index, were normal. The spirometry showed moderate restriction due to collapse of the right middle and lower lobes. We also performed an overnight type 1 polysomnography. There was no evidence of sleep disordered breathing or hypoventilation. Arterial blood gas analysis was also performed to look for hypoventilation. It showed $\mathrm{pH}$ of 7.41, arterial carbon dioxide tension of $42 \mathrm{mmHg}$, arterial oxygen tension of $85 \mathrm{mmHg}$ and bicarbonate level of $25 \mathrm{mEq} \cdot \mathrm{L}^{-1}$.

\section{Task 5}

What is the ideal management option?
a. Tracheobronchial stent
b. Surgery
c. Observation and conservative management
d. Positive airway pressure therapy 


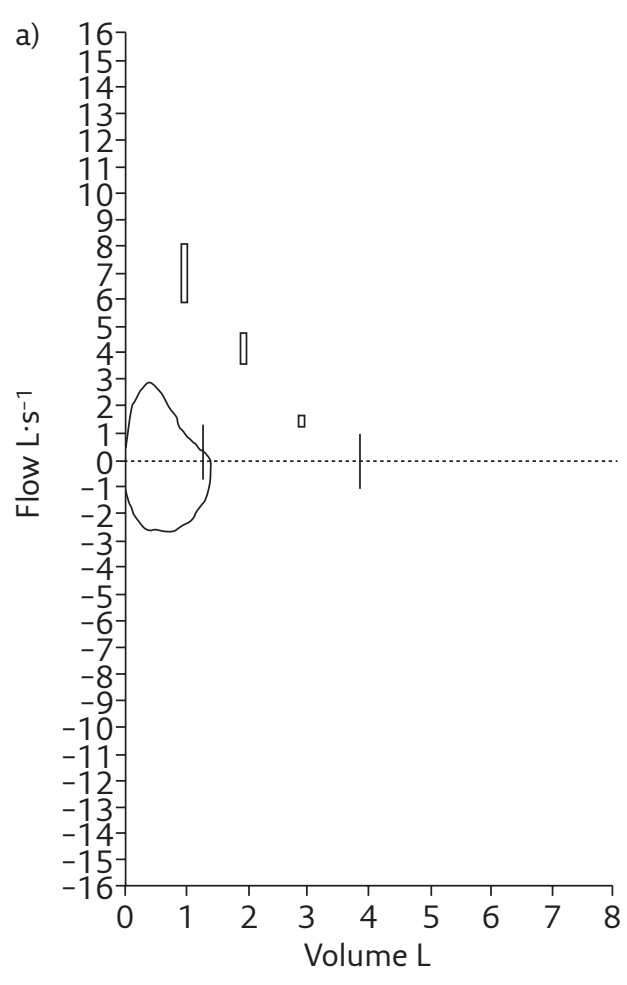

Figure 6 a) Flow-volume loop; b) volume-time graphic.

\section{Answer 5 \\ c. Observation and conservative management}

The management options for ECAC include all those in task 5. A conservative approach with medical treatment and noninvasive positive pressure ventilation is preferred over potentially harmful open surgical procedures [2]. Continuous positive airway pressure (CPAP) is the first line treatment of ECAC in symptomatic patients. It acts as a pneumatic splint and improves both pulmonary functions and quality of life [10]. CPAP improves dyspnoea, cough and secretion in selected patients with ECAC [2]. In addition to these, chest physiotherapy and mucolytics help in managing secretions. Tracheobronchial stent followed by surgery is the definitive management of ECAC but this intervention is necessary only if the patient has a significant impairment of functional status, a poor quality of life and $>95 \%$ tracheobronchial collapse [2, 10-12]. Our patient was relatively asymptomatic and was detected to have EDAC incidentally, and hence was managed conservatively.

\section{Discussion}

Parkinsonian disorders refer to a group of neurological disorders characterised by movementrelated abnormalities. Parkinson's disease is the most common cause. Other causes include multiple-system atrophy, progressive supranuclear

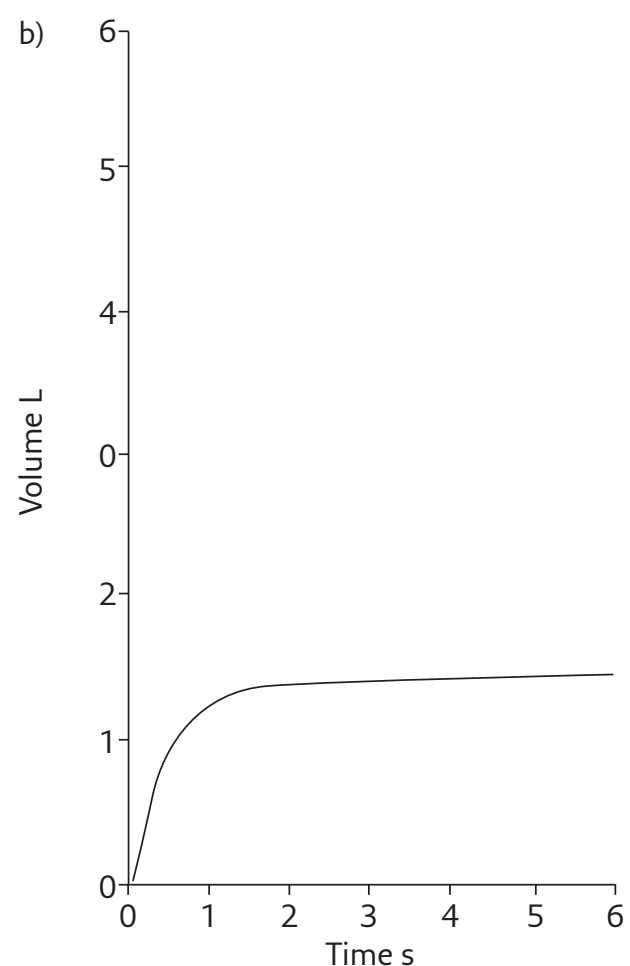

Table 1 Spirometry findings of the patient

\begin{tabular}{|c|c|c|c|c|}
\hline Parameter & Predicted & LLN & Observed & $\%$ predicted \\
\hline FVC L & 3.79 & 2.79 & 1.39 & 37 \\
\hline FEV $_{1} \mathrm{~L}$ & 3.01 & 2.17 & 1.25 & 41 \\
\hline $\mathrm{FEV}_{1} / \mathrm{FVC} \%$ & 86.14 & 74.35 & 89.85 & 104 \\
\hline PEF L-s $\mathbf{s}^{-1}$ & 7.91 & 5.92 & 2.90 & 37 \\
\hline PIF L·s $\mathbf{s}^{-1}$ & & & 2.64 & \\
\hline MEF L.s $\mathbf{s}^{-1}$ & 3.40 & 1.69 & 1.85 & 54 \\
\hline $\mathrm{MEF}_{50} \mathrm{~L} \cdot \mathrm{s}^{-1}$ & 4.17 & 2.02 & 2.01 & 48 \\
\hline $\mathrm{FIF}_{50} \mathrm{~L} \cdot \mathrm{s}^{-1}$ & & & 2.62 & \\
\hline Empey's index & & & 6.9 & \\
\hline Miller and Hyatt index & & & 0.76 & \\
\hline
\end{tabular}

LLN: lower limit of normal; FVC: forced vital capacity; FEV1: forced expiratory volume in $1 \mathrm{~s}$; PEF: peak expiratory flow; PIF: peak inspiratory flow; MEF: mean mid-expiratory flow from $25-75 \%$ of FVC; $M_{50}$ : mid-expiratory flow at $50 \%$ of FVC; FIF $_{50}$ : forced inspiratory flow at $50 \%$ FVC; Empey's index: $\mathrm{FEV}_{1} / \mathrm{PEF}$; Miller and Hyatt index: $\mathrm{FEF}_{50} / \mathrm{FIF}_{50}$.

palsy and corticobasal degeneration [13]. Our patient was diagnosed to have the progressive supranuclear palsy type of parkinsonian disorder by a neurologist.

Parkinsonian disorders have motor features; namely, muscle rigidity, tremors at rest, bradykinesia and postural instability. Nonmotor involvement leading to dysphagia and constipation has also been reported [14]. Parkinsonian disorders are known to cause muscle atrophy; however. tracheobronchial 
muscle atrophy has not been documented so far [15]. Only one case has been reported in the past with parkinsonian disorder, EDAC and asthma [16]. Currently, there is no evidence to support a causal relationship between parkinsonian disorders and EDAC; however, studies are required to establish of refute this relationship. Obstructive sleep apnoea was thought to be more common in parkinsonian disorders due to higher association of upper airway obstruction. However, studies have shown conflicting evidence regarding obstructive sleep apnoea in parkinsonian disorders $[17,18]$. Our patient did not have obstructive sleep apnoea.

Bronchoscopy is the gold standard to confirm a diagnosis of ECAC as it allows real-time visualisation and characterisation of the airways [19]. A decrease in the size of the lumen by $\leq 70 \%$ during exhalation is regarded as normal, between $70 \%$ and $80 \%$ as mild obstruction, between $81 \%$ and $90 \%$ as moderate obstruction, and between $91 \%$ and $100 \%$ as severe obstruction [6]

A classification of EDAC based on objective quantifiable criteria has been developed, and can be applied before and after therapeutic interventions to document not only the changes in the extent and severity of collapse but also the impact of these changes on functional class [2]. Five domains are addressed [2]:

- functional class

- extent

- morphology
- origin

- severity of airway collapse

Though all the domains are important, dyspnoea is the most consistent metric of surgical treatment [3]. A Y-shaped, silicone tracheobronchial stent is placed in advance for patient assessment regarding candidacy for surgical intervention. The stent is deployed within the airway portions that would be stabilised by a surgical tracheobronchoplasty. After a 2-week trial, patients are evaluated for the level of symptomatic improvement attributable to stabilisation of the central airways and are subjected to surgery.

\section{Conclusion}

Combined collapse of right middle and lower lobe is difficult to detect on chest radiograph, and requires CT scan for confirmation. ECAC can rarely lead to lobar collapse. ECAC is classified into four types based on the morphology of the airways during inspiration and expiration: crescent-type TBM, sabre sheath-type TBM, circumferential-type TBM and EDAC. Bronchoscopy is the gold standard for confirming ECAC. A substantial number of patients with moderate to severe ECAC can demonstrate normal flow-volume loops. Surgical intervention for tracheobronchomalacia is required only if the collapse is $95-100 \%$ and the patient is symptomatic.

Affiliations

Dipti Gothi, Mahismita Patro, Mohit Agarwal, Sameer Vaidya

Dept of Pulmonary Medicine, ESI PGIMSR, New Delhi, India.

\section{Conflict of interest}

None declared

\section{References}

1. Lee KS, Logan PM, Primack SL, et al. Combined loba atelectasis of the right lung: imaging findings. AJR 1994; 163: 43-47

2. Murgu S, Colt H. Tracheobronchomalacia and excessive dynamic airway collapse. Clin Chest Med 2013; 34: 527-555.

3. Buitrago DH, Wilson JL, Parikh M, et al. Current concepts in severe adult tracheobronchomalacia: evaluation and treatment. J Thorac Dis 2017; 9: E57-E66.

4. Lomasney L, Bergin CJ, Lomasney J, et al. CT appearance of lunate trachea. J Comput Assist Tomogr 1989; 13: 520-522.

5. Boiselle PM, Ernst A. Tracheal morphology in patients with tracheomalacia: prevalence of inspiratory lunate and expiratory "frown" shapes. J Thorac Imaging 2006; 21: 190-196.

6. Banka R, Terrington D, Kamath AV. A 31-year-old female with a rare cause of recurrent lower lobar collapses. Breathe 2018 14: e72-e77.

7. Marini T, Hobbs SK, Chaturvedi A, et al. Beyond bronchitis: a review of the congenital and acquired abnormalities of the bronchus. Insights Imaging 2017; 8: 141-153.
8. Ridge CA, O'donnell CR, Lee EY, et al. Tracheobronchomalacia: current concepts and controversies. J Thorac Imaging 2011; 26: 278-289.

9. Majid A, Sosa AF, Ernst A, et al. Pulmonary function and flowvolume loop patterns in patients with tracheobronchomalacia. Respir Care 2013; 58: 1521-1526.

10. López-Padilla D, García-Luján R, Maestu LP, et al Tracheobronchomalacia treatment: how far have we come? J Thorac Dis 2016; 8: 3490-3493.

11. Ernst A, Majid A, Feller-Kopman D, et al. Airway stabilization with silicone stents for treating adult tracheobronchomalacia: a prospective observational study. Chest 2007; 132: 609-616.

12. Majid A, Guerrero J, Gangadharan S, et al. Tracheobronchoplasty for severe tracheobronchomalacia: a prospective outcome analysis. Chest 2008; 134: 801-807.

13. Dickson DW. Parkinson's Disease and Parkinsonism: Neuropathology. Cold Spring Harb Perspect Med 2012; 2 a009258. 
14. Sauerbier A, Chaudhuri KR. Non-motor symptoms: the core of multi-morbid Parkinson's disease. BrJ Hosp Med 2014; 75: 18-24.

15. Cano-de-la-Cuerda R, Pérez-de-Heredia M, Miangolarra-Page JC, et al. Is there muscular weakness in Parkinson's disease? Am J Phys Med Rehabil 2010; 89: 70-76.

16. Harada Y, Kondo T. Excessive dynamic airway collapse detected using nondynamic CT. Intern Med 2016; 55 1477-1479.
17. Zeng J, Wei M, Li T, et al. Risk of obstructive sleep apnea in Parkinson's disease: a meta-analysis. PLOS ONE 2013; 8: e82091.

18. Crosta F, Desideri G, Marini C. Obstructive sleep apnea syndrome in Parkinson's disease and other parkinsonisms. Funct Neurol 2017; 32: 137-141.

19. Majid A, Gaurav K, Sanchez JM, et al. Evaluation of tracheobronchomalacia by dynamic flexible bronchoscopy. A pilot study. Ann Am Thorac Soc 2014; 11: 951-955. 